\title{
Differential expression profile study and gene function analysis of maternal foetal-derived circRNA for screening for Down's syndrome
}

\author{
WEIGUO SUI ${ }^{1,2^{*}}$, QING GAN $^{1,2^{*}}$, YAN CHANG $^{3}$, MINGLIN OU $^{1,2}$, JIEJING CHEN $^{1,2}$,

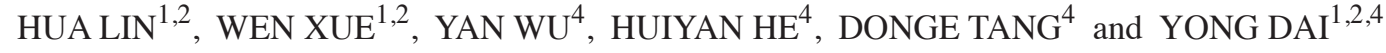 \\ ${ }^{1}$ Guangxi Key Laboratory of Metabolic Diseases Research; ${ }^{2}$ Kidney Diseases Research, \\ Department of Nephrology; ${ }^{3}$ Reproductive Center, Guilin No. 924 Hospital, Guilin, Guangxi 541002; \\ ${ }^{4}$ Clinical Medical Research Center, The Second Clinical Medical College of Jinan University, \\ Shenzhen People's Hospital, Shenzhen, Guangdong 518020, P.R. China
}

Received November 21, 2018; Accepted October 8, 2019

DOI: $10.3892 /$ etm.2019.8288

\begin{abstract}
Recent studies have shown that circular RNAs (circRNAs) exhibit differential expression in certain diseases. However, to the best of our knowledge, maternal fetal-derived circRNAs and mRNAs associated with Down's syndrome (DS) have not yet been investigated. A total of 12 umbilical cord blood samples were collected from pregnant women, including six women carrying fetuses with DS (diagnosed by G-banding karyotype analysis), and six women carrying fetuses without DS. In addition, 12 peripheral blood samples were obtained from children, including six children with DS and six children without DS. Gene chip technology was used to screen for differentially expressed circRNAs and mRNAs in the cord blood samples, and were subsequently verified by reverse transcription-quantitative polymerase chain reaction in peripheral blood from the children to identify potential biomarkers. Furthermore, circRNA/microRNA (miRNA) interactions were predicted using Arraystar miRNA target prediction software. There was a significant difference in the expression of hsa_circRNA_103127, hsa_circRNA_103112 and hsa_circRNA_104907 between cord blood obtained from the women carrying fetuses with and without DS, and between peripheral blood obtained from children with and without DS $(\mathrm{P}<0.01)$. As hsa_circRNA_103112 exhibited significant
\end{abstract}

Correspondence to: Professor Yong Dai or Dr Donge Tang, Clinical Medical Research Center, The Second Clinical Medical College of Jinan University, Shenzhen People's Hospital, 1017 Dongmen North Road, Shenzhen, Guangdong 518020, P.R. China

E-mail: daiyong22@aliyun.com

E-mail: donge66@126.com

*Contributed equally

Key words: circular RNA, Down's syndrome, maternal, fetal, differential expression, gene function differences in expression between cord blood obtained from the women carrying fetuses with and without DS and between peripheral blood obtained from children with and without DS, its corresponding gene, ubiquitin specific peptidase 25 , may be involved in the pathogenesis of the condition. These results suggested that hsa_circRNA_103112 may be upregulated in individuals with DS, resulting in an expression imbalance of diploid genes through interactions among circRNA, miRNA and mRNA. Therefore, the level of hsa_circRNA_103112 in the peripheral blood of a pregnant woman may serve as potential biomarker of fetal DS during non-invasive prenatal screening.

\section{Introduction}

Down's syndrome (DS), or trisomy 21, is caused by the presence of three copies of chromosome 21 and is the most common genetic cause of intellectual disability (1) DS has an incidence of up to 1 in 700 live births (2), and this incidence increases with increasing maternal age (3). DS is characterized by mild-to-moderate learning disabilities, craniofacial abnormalities and hypotonia (4), and is typically accompanied by more than 80 diseases such as congenital heart disease, low immunity, leukaemia (5). DS is associated with financial burden on the family and society, and there is no effective therapy for the chromosomal abnormalities. Traditional prenatal screening and diagnostic methods are associated with several shortcomings, and the clinical application of novel non-invasive prenatal diagnostic methods are restricted by various factors (6). Therefore, there is a requirement for the development of an efficient, accurate and cost-effective screening method.

Circular RNAs (circRNAs) are a type of RNA, which unlike linear RNA, have joined 5' and 3'ends that form a continuous loop (7), a structure that provides resistance against exonucleases and further differentiates them from other types of RNA (8). circRNAs are a class of endogenous noncoding RNAs produced by the non-canonical form of alternative splicing $(7,9)$. Developments in bioinformatics analysis and 
RNA deep sequencing technology revealed that circRNAs are abundant, conserved and stable in mammalian cells (10-16). Previous studies demonstrated that certain circRNAs not only act as competing endogenous RNA for gene expression regulation, but also repress the function of microRNAs (miRNAs/miRs) by binding to miRNA response elements (MREs), thereby regulating the expression level of other related RNAs $(13,17,18)$. Studies have also shown that imbalances in the expression of certain miRNAs may lead to extensive changes in mRNA and protein expression, and play an important role in the development and progression of disease $(19,20)$. In addition, certain circRNAs serve as a template during translation and guide the synthesis of proteins $(21,22)$. Moreover, circRNAs bind with specific proteins to inhibit or regulate protein activity $(23,24)$. Furthermore, certain circRNAs may participate in development of disease, particularly in cancer. circRNAs exhibit diverse functions and may serve as diagnostic or predictive biomarkers of disease $(25,26)$ and novel therapeutic targets. Sun et al (27) revealed that hsa circ_0000520 may be involved in the development of gastric cancer and may therefore serve as a biomarker of the disease. Yang et al (28) demonstrated that differentially expressed circ-F-box, WD repeat domain containing 7 (FBXW7) and FBXW7-185aa have potential prognostic significance in brain cancer. However, to the best of our knowledge, the association between circRNAs and DS has not been previously reported. Therefore, it is of significance to carry out an in-depth and systematic research on circRNA of DS.

\section{Materials and methods}

Samples. Umbilical cord blood samples were obtained from 12 pregnant women, six (age range, 35-43 years; mean age, 39.7 years) carrying fetuses with DS (two male and four female) and six (27-36 years, 31.3 years of mean age) carrying fetuses without DS (two male and four female) at a gestational age of 18-22 weeks. The participants were recruited at Shenzhen People's Hospital (Shenzhen, China) between January 2013 and December 2014. Diagnosis of fetal DS was performed by chromosomal examination. In addition, peripheral blood samples were obtained from 12 children, six with DS and six without DS (two males and four females; age range, 5-12 years, mean age, 8 years) at Guilin No. 924 Hospital (Guilin, China) between January 2015 and December 2016. The present study was approved by the Ethics Committees of Shenzhen People's Hospital (Shenzhen, China) and Guilin No. 924 Hospital (Guilin, China). Written informed consent was obtained from all pregnant women and the parents/guardians of the children.

Sample processing. Ultrasound-guided umbilical cord blood extraction was performed and a total of $3 \mathrm{ml}$ extracted blood was placed in EDTA-containing anticoagulant tubes. Peripheral blood $(2 \mathrm{ml}$ ) of 12 children was extracted with a needle and placed in EDTA-containing anticoagulant tubes. According to the single nuclear cell extraction protocol, $1 \mathrm{ml}$ umbilical cord blood mononuclear cells (PBMCs) was extracted using lymphocyte separation solution (MD Pacific Biotechnology Co., Ltd.), mixed with $1 \mathrm{ml}$ TRIzol ${ }^{\circledR}$ reagent (Invitrogen; Thermo Fisher Scientific, Inc.), placed in cryopreservation tubes and stored at $-80^{\circ} \mathrm{C}$ until further use.
Extraction of total RNA. The frozen PBMCs were thawed at room temperature and total RNA was extracted using TRIzol ${ }^{\circledR}$ reagent according to the manufacturer's instructions. Total RNA was subsequently treated with DNase I to remove genomic DNA contamination. The purity and concentration of the RNA were determined using a spectrophotometer (NanoDrop ND-1000; Thermo Fisher Scientific, Inc.). RNA integrity was assessed using standard denaturing agarose gel electrophoresis (1\%).

circRNA labelling and array hybridization. Total RNA from each sample was amplified and transcribed into fluorescent cRNA utilizing random primers according to the Arraystar Super RNA Labeling protocol (Arraystar, Inc.). The labelled cRNAs were hybridized onto Arraystar Human circRNA arrays (cat. no. 6x7K; Arraystar, Inc.) and incubated for $17 \mathrm{~h}$ at $65^{\circ} \mathrm{C}$ in an Agilent hybridization oven (Agilent Technologies, Inc.). After washing, slides were scanned with the Axon GenePix 4000B Scanner (Molecular Devices, LLC).

circRNA data collection and analysis. Scanned images were imported into GenePix Pro software (version 6.0; Molecular Devices, LLC) for grid alignment and raw data extraction. Quantile normalization of raw data and subsequent data processing were performed using the $\mathrm{R}$ software package heatmap.2 (version 3.2.0; https://mirrors.tuna.tsinghua.edu. $\mathrm{cn} / \mathrm{CRAN} /$ ). The circRNAs that at least 1 out of 2 samples had flagged as 'expressed' (greater than 2 times background standard deviation) were retained for further differential analyses. Differentially expressed circRNAs between umbilical cord blood samples obtained from pregnant woman carrying fetuses with DS and without DS were identified through fold-change ( $F C \geq 2.0$ or $\leq-2.0$ ) or volcano plot filtering.

$m R N A$ labelling and array hybridization. In the present study, sample labelling and array hybridization were performed according to the Agilent One-Color Microarray-Based Gene Expression Analysis protocol (Agilent Technologies, Inc.). Briefly, total RNA from each sample was linearly amplified and labelled with Cy3-UTP (Enzo Life Sciences, Inc.). The labelled cRNAs were purified using an RNeasy Mini kit (Qiagen, Inc.). The concentration and specific activity of the labelled cRNAs (pmol Cy3/ $\mu \mathrm{g}$ cRNA) were measured using a spectrophotometer. A total of $1 \mu \mathrm{g}$ of each labelled cRNA was fragmented by the addition of $11 \mu \mathrm{l} 10 \mathrm{X}$ blocking agent (LMAI Bio) and $2.2 \mu 125 \mathrm{X}$ fragmentation buffer (Agilent Technologies, Inc.), then heated at $60^{\circ} \mathrm{C}$ for $30 \mathrm{~min}$. Subsequently, $55 \mu 12 \mathrm{X}$ GEx Hybridization Buffer HI-RPM (Agilent Technologies, Inc.) were added to dilute the labelled cRNA and $100 \mu 1$ hybridization solution (Agilent Technologies, Inc.) were dispensed into the gasket slide and assembled on the gene expression microarray slide. The slides were incubated for $17 \mathrm{~h}$ at $65^{\circ} \mathrm{C}$ in the aforementioned Agilent hybridization oven. The hybridized arrays were washed, fixed and scanned using the Agilent DNA Microarray Scanner (cat. no. G2505C; Agilent Technologies, Inc.).

Analysis and functional analysis of differentially expressed genes. The differentially expressed genes ( $\mathrm{FC} \geq 2.0$ or $\leq-2.0)$ were selected using Agilent GeneSpring GX software (version 12.1; Agilent Technologies, Inc.). Then, hierarchical clustering was performed using scripts prepared by Aksomics 
Inc. Gene Ontology (GO; http://www.geneontology.org) analysis and Kyoto Encyclopedia of Genes and Genomes (KEGG; https://www.genome.jp/kegg/) pathway analysis were performed using standard enrichment calculation methods.

mRNA data analysis. Agilent Feature Extraction software (version 11.0.1.1; Agilent Technologies, Inc.) was used to analyse the acquired array images. Quantile normalization and subsequent data processing were performed using the GeneSpring GX software package (version 12.1; Agilent Technologies, Inc.). After quantile normalization of the raw data, genes in which at least 1 out of 2 samples had flags in Detected ('All Targets Value') were chosen for further data analysis. Differentially expressed genes between the umbilical cord blood samples obtained from pregnant woman carrying fetuses with and without DS were identified through FC and volcano plot filtering. Hierarchical clustering was performed using the aforementioned $\mathrm{R}$ scripts. GO and KEGG analyses were performed using standard enrichment calculation methods.

Validation of differentially expressed circRNA and $m R N A$ by reverse-transcription quantitative $P C R(R T-q P C R)$. Six circRNAs (Table I) and three mRNAs (Table II) were randomly selected to verify the accuracy of the results in peripheral blood samples of children with and without DS by RT-qPCR. Total RNA was extracted from the samples by TRI REAGENT BD (Molecular Research Center, Inc.) and reverse transcribed into cDNA using SuperScriptTM III Reverse Transcriptase (Invitrogen; Thermo Fisher Scientific, Inc.), 5X RT buffer, $10 \mathrm{mM}$ dNTP mixture (dATP, dGTP, dCTP and dTTP; $2.5 \mathrm{mM}$ each; HyTest Ltd.) and random primers supplied by Ying jun Biotechnology Co., Ltd., at $65^{\circ} \mathrm{C}$ for $5 \mathrm{~min}$ and on ice for $2 \mathrm{~min}$. qPCR was subsequently performed with 2X SYBRGreen PCR master mix (Arraystar, Inc.) and primers (Table III). The following thermocycling conditions were used: $95^{\circ} \mathrm{C}$ for $5 \mathrm{~min}$ followed by 40 cycles of a denaturing step at $95^{\circ} \mathrm{C}$ for $10 \mathrm{sec}$ and an annealing/extension step at $60^{\circ} \mathrm{C}$ for $60 \mathrm{sec}$. All reactions were run in triplicate. mRNA levels were quantified using the $2^{-\Delta \Delta C q}$ method (29) and normalized to the internal reference gene $\beta$-actin.

Prediction of circRNA/microRNA interaction. To identify circRNAs acting as miRNA sponges, the circRNA/miRNA interactions were predicted using a miRNA target prediction software (cat. no. AS-S-CR-H-V2.0; Arraystar, Inc.) based on TargetScan (30) and miRanda (31), and the differentially expressed circRNAs were annotated with the circRNA/miRNA interaction information.

Statistical analysis. All statistical data were analysed using SPSS software (version 19.0). The statistical difference between the two groups was analysed using a Student's t-test. $\mathrm{P}<0.05$ was considered to indicate a statistically significant difference.

\section{Results}

Differential expression analysis of circRNA in umbilical cord blood. circRNAs exhibiting a $\mathrm{FC} \geq 2.0$ or $\leq-2.0$ were identified as being differentially expressed between umbilical cord blood samples of pregnant woman carrying fetuses with and without
Table I. Verification of the circRNA microarray in peripheral blood samples obtained from children with and without Down's syndrome.

\begin{tabular}{lccc}
\hline circRNA & Fold-change & $2^{-\Delta \mathrm{CC}}$ value & P-value \\
\hline hsa_circRNA_103135 & 4.49 & 1.23 & 0.4250 \\
hsa_circRNA_103127 & 2.64 & 0.46 & 0.0009 \\
hsa_circRNA_103112 & 2.04 & 0.42 & 0.0002 \\
hsa_circRNA_103137 & -2.16 & 1.34 & 0.1530 \\
hsa_circRNA_104907 & -4.51 & 3.29 & $1 \times 10^{-6}$ \\
hsa_circRNA_101116 & -5.07 & 1.17 & 0.3180 \\
\hline
\end{tabular}

circRNA, circular RNA.

DS. A total of 735 differentially expressed circRNAs were detected between the two groups, of which 414 were upregulated and 321 were downregulated (Table SI). The circRNA expression profile in the two groups is presented in Fig. 1A.

A scatter-plot was used to visualize the variation (or reproducibility) of circRNA expression between two groups of samples. The values of the $x-$ and $y$-axes in the scatter-plot were the normalized signal values of the samples ( $\log 2$ scaled) or the averaged normalized signal values of groups of samples ( $\log 2$ scaled). The green lines represented FCs. The circRNAs above the top green line and below the bottom green line indicated a $\geq 2.0-\mathrm{FC}$ in circRNA expression between the two groups.

Differential expression analysis of mRNA in umbilical cord blood samples. mRNAs with a $\mathrm{FC} \geq 2.0$ or $\leq-2.0$ were selected as the differentially expressed ones. In the umbilical cord blood samples obtained from pregnant woman carrying fetuses with and without DS, 6,619 differentially expressed genes were detected, of which 3,411 and 3,208 genes were upregulated and downregulated, respectively (Table SII). The mRNA expression profile of the two groups is shown in Fig. 1B.

$R T-q P C R$ to validate circRNA expression. A total of six differentially expressed circRNAs in the microarray experiments were randomly selected and the relative expression was calculated using the $2^{-\triangle \Delta C q}$ method (29). The circRNAs included three upregulated circRNAs (hsa_circRNA_103135, hsa_circRNA_103127 and hsa_circRNA_103112) and three downregulated circRNAs (hsa_circRNA_103137, hsa_circRNA_104907 and hsa_circRNA_101116). The results were validated using peripheral blood samples obtained from children with and without DS (Table I).

As seen in Table I, there were no significant differences in the expression levels of hsa_circRNA_103135, hsa_circRNA_103137 and hsa_circRNA_101116 (P>0.05), but there were extremely significant differences in the expression levels of hsa_circRNA_103127, hsa_circRNA_103112 and hsa_circRNA_104907 $(\mathrm{P}<0.01)$ between six children with DS and six children without DS.

$R T-q P C R$ to validate the differentially expressed genes. A total of three statistically significant genes [dual specificity 
Table II. Verification of differentially expressed genes in peripheral blood samples obtained from children with and without Down's syndrome.

\begin{tabular}{|c|c|c|c|}
\hline Gene & Fold-change & $2^{-\Delta \Delta C q}$ value & P-value \\
\hline Dual specificity tyrosine phosphorylation regulated kinase $1 \mathrm{~A}$ & 2.64 & 1.35 & 0.190 \\
\hline Ubiquitin specific peptidase 25 & 2.04 & 0.84 & 0.009 \\
\hline Spermatid perinuclear RNA binding protein & -4.51 & 1.35 & 0.150 \\
\hline
\end{tabular}

Table III. Primers used for PCR.

Primer sequence $\left(5^{\prime} \rightarrow 3^{\prime}\right)$

Gene

Forward

Reverse

$\beta$-actin (Human)

hsa_circRNA_103135

hsa_circRNA_103127

hsa_circRNA_103112

hsa_circRNA_103137

hsa_circRNA_104907

hsa_circRNA_101116

USP25

DYRK1A

STRBP

GTGGCCGAGGACTTTGATTG
GGAGGGCTTCTACGTCATCTTC
GACCGTCGCCAGCCAAAC
GCACTTCCTGGCAATGATAGAT
ATCCTGTCCTCCTAAACCTCCA
TACAAAAGAGCCCACGCTAACT
ACTGCCTACTGCTATAATTCTGAA
CCTGTTGACGATATTGACGCTAG
CAGGTCCAGAGTATGAGTGC
GACACTCCACTCTGACACCCTC

GTGGCCGAGGACTTTGATTG

GGAGGGCTTCTACGTCATCTTC

GCACTTCCTGGCAATGATAGAT

ATCCTGTCCTCCTAAACCTCCA

TACAAAAGAGCCCACGCTAACT

CCTGTTGACGATATTGACGCTAG

GACACTCCACTCTGACACCCTC
CCTGTAACAACGCATCTCATATT
GTCTATGTAGGAGTGCGGGGTT
GAGTCCAGCGGCAAAACTATAA
GGCTTGCTGTAGTATCTGGGTG
TCTCGCTGACCAAGAACTGAATA
TGTCTGAAGGCTTGTTCTCTGG
GTTGTTTCTGGGCTTCTGTGAG
CTCCCTGTTGTTCTGTTGTGCT
GGCAGCGTAATCTCAACAC
CTCCCTGACAAGAAACTATGCTAA

circRNA, circular RNA; USP25, ubiquitin specific peptidase 25; DYRK1A, dual specificity tyrosine phosphorylation regulated kinase 1A; STRBP, spermatid perinuclear RNA binding protein.
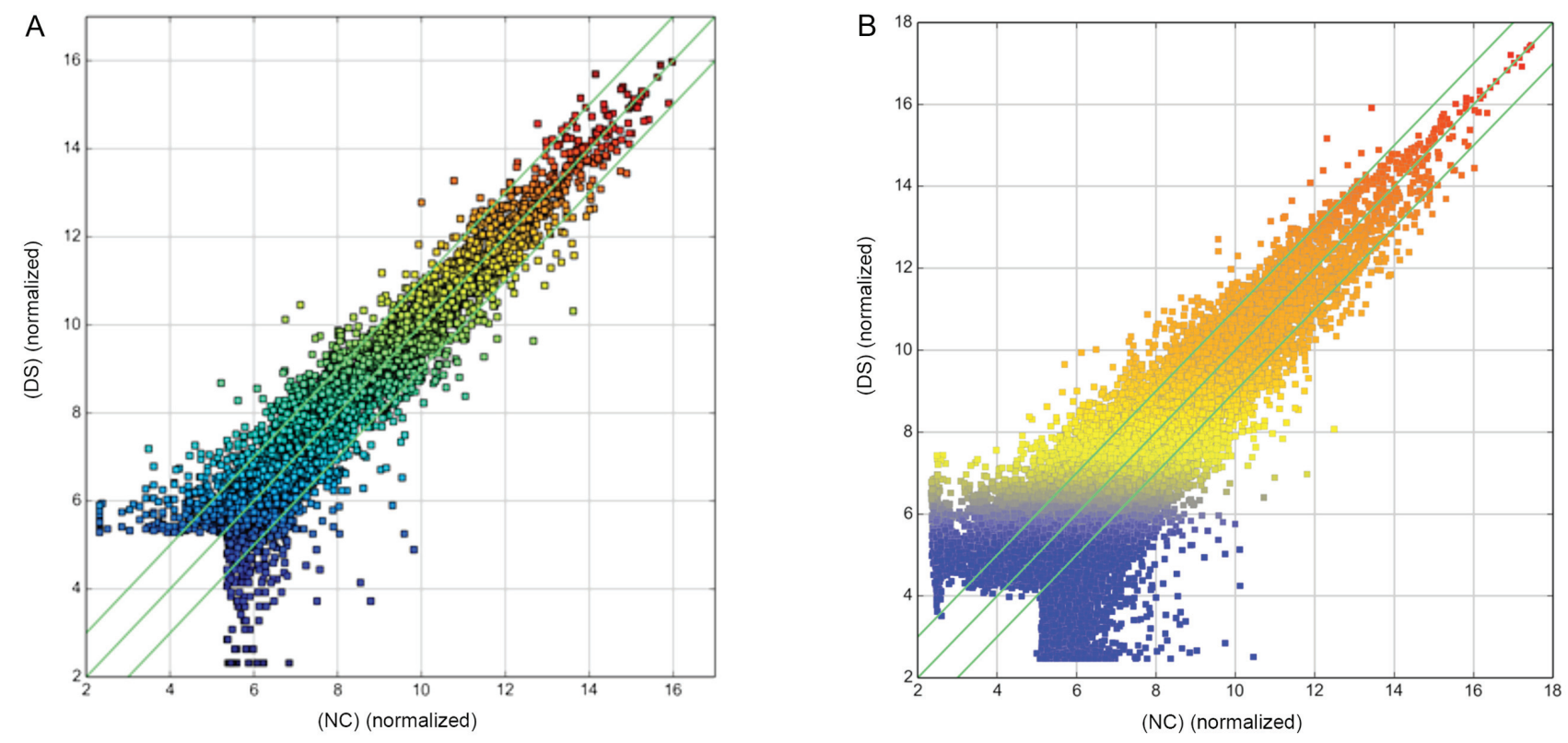

Figure 1. Scatter plots of differentially expressed circRNA and mRNA. (A) Scatter plot of differentially expressed circRNA of women carrying fetuses with DS and women carrying fetuses without DS. (B) Scatter plot of differentially expressed mRNA of women carrying fetuses with DS and women carrying fetuses without DS. The higher the index signal value of the $\mathrm{X}$ and $\mathrm{Y}$ axes detected in the scatter plot, the darker the color. Absolute value of FC between the two samples was $>2.0$ for data above the top green line and below the bottom green.

tyrosine phosphorylation regulated kinase 1A (DYRK1A), ubiquitin specific peptidase 25 (USP25) and spermatid perinuclear RNA binding protein (STRBP)], corresponding to three circRNAs (hsa_circRNA_103127,hsa_circRNA_103112 

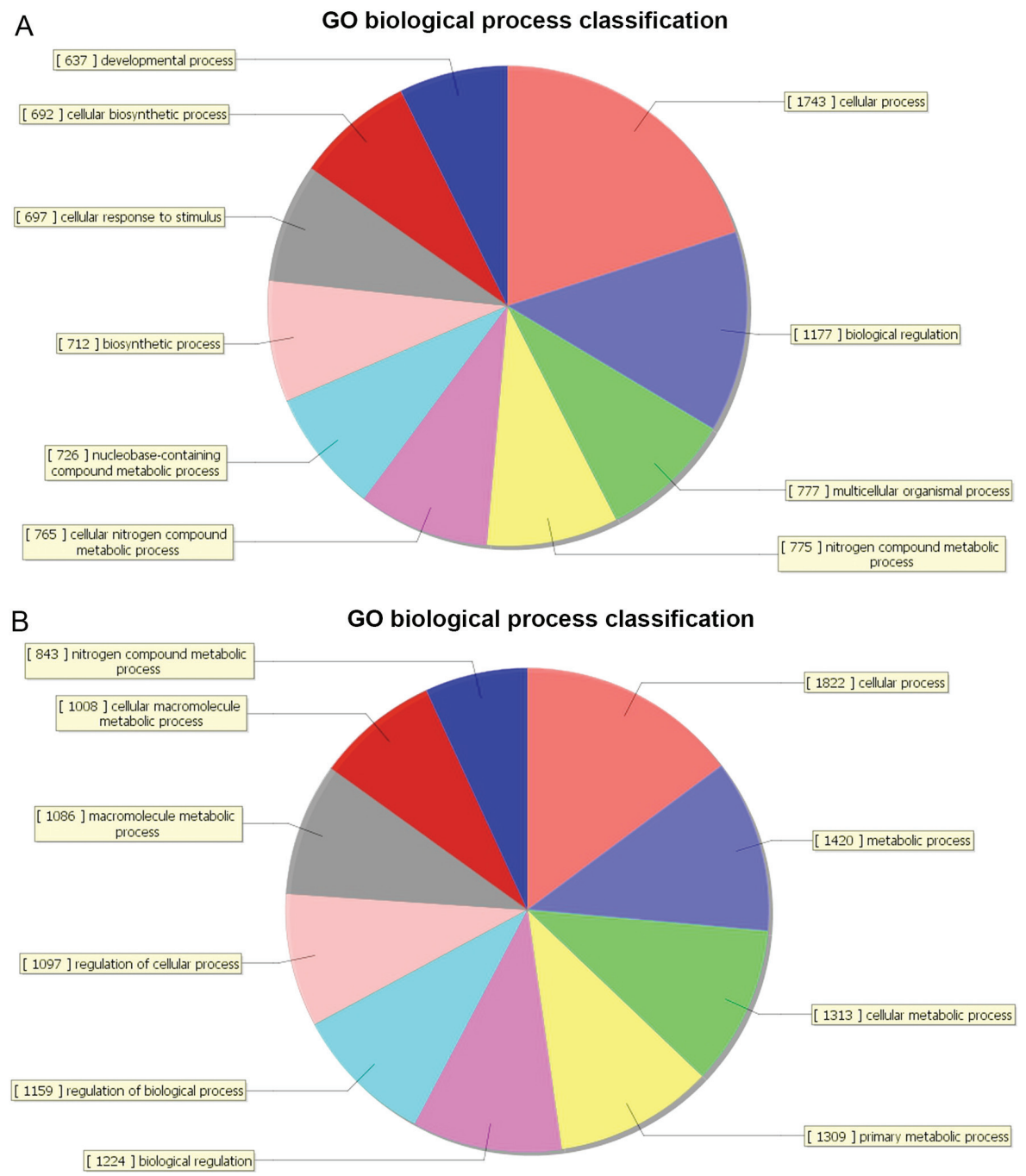

Figure 2. GO biological process classification. (A) GO biological process classification of upregulated genes. (B) GO biological process classification of downregulated genes. GO, gene ontology.

and hsa_circRNA_104907) were validated in peripheral blood samples obtained from children with and without DS. The results are shown in Table II.

As seen in Table II, there was no significant difference in the expression levels of DYRK1A and STRBP $(\mathrm{P}>0.05)$, but there was extremely significant difference in the expression level of USP25 $(\mathrm{P}<0.01)$ between six children with DS and six children without DS.

$G O$ analysis of the differentially expressed genes. GO covers three domains: Biological process, cellular component and molecular function. GO analysis of the differentially expressed genes revealed that 805 genes were associated with the biological process domain, of which 315 were upregulated (Fig. 2A) and 490 were down-regulated (Fig. 2B). The five most enriched biological process terms were 'cellular processes', 'primary metabolic processes', 'cellular metabolic processes', 'biological regulation' and 'nitrogen compound metabolic processes'. A total of 189 genes were associated with the cell composition domain, of which 66 were upregulated (Fig. 3A) and 123 genes were downregulated (Fig. 3B). The five most enriched cell composition terms were 'intracellular', 'intracellular part', 'organelle', 'intracellular organelle' and 'membrane-bound organelle'. A total of 155 genes were associated with the molecular function domain, of which 77 were upregulated (Fig. 4A) and 78 were down-regulated (Fig. 4B). The five most enriched molecular function terms were 'binding', 'protein binding', 'RNA binding', 'ligase activity' and 'catalytic activity'.

Pathway analysis of the differentially expressed genes. Pathway analysis of the differentially expressed genes allows the identification of genes related to specific cell pathways. Pathway analysis revealed that the differentially expressed genes were significantly enriched in 73 pathways (Fig. 5A and B). The upregulated genes were involved in 23 pathways and the downregulated genes were involved in 50 pathways. Differentially expressed genes were mainly involved in 'adhesive spots', 'ECM-receptor interactions', 'gap junctions', 'protein digestion and absorption', 'vitamin digestion and absorption' and 'TNF signalling pathway'. Additional pathways included 'primary immunodeficiency', 'systemic lupus erythematosus', 'rheumatoid arthritis', 'type I diabetes 
A

GO cellular component classification

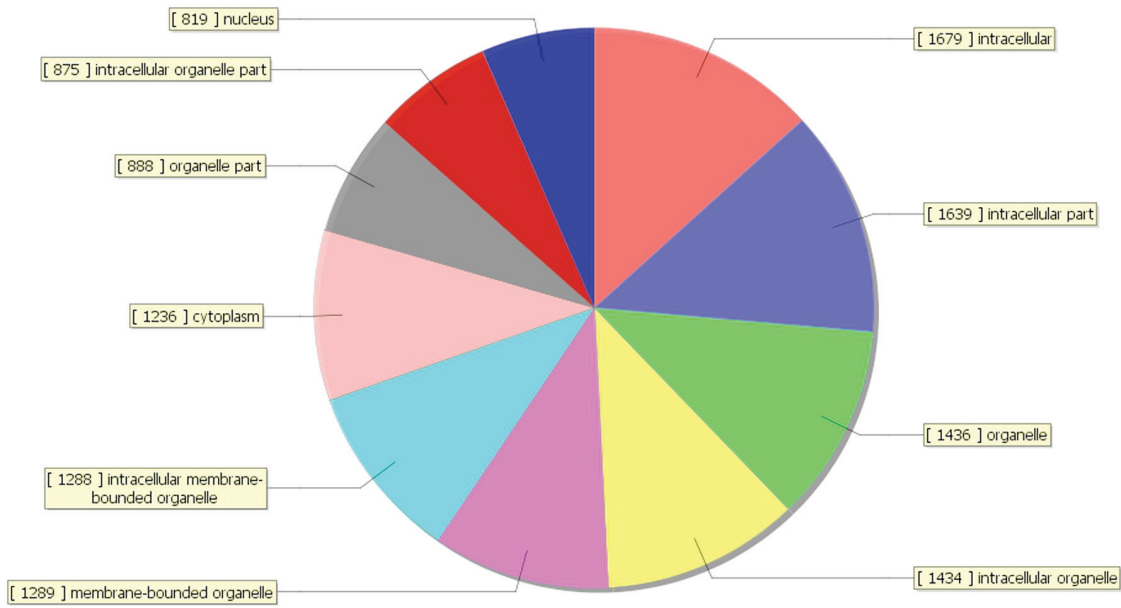

B

GO cellular component classification

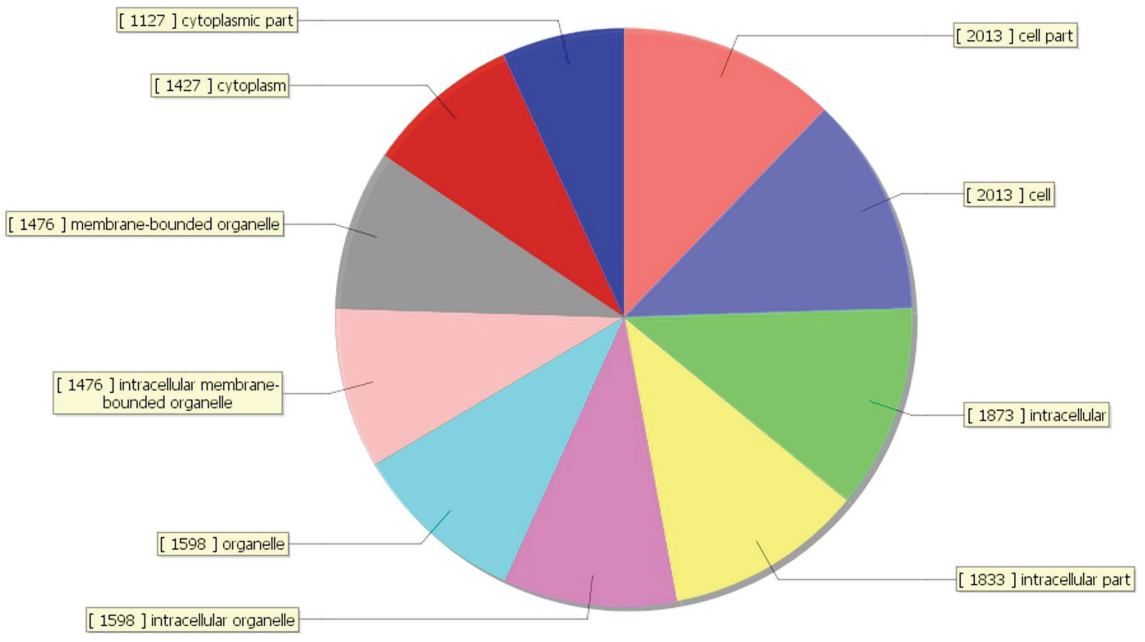

Figure 3. GO cellular component classification. (A) GO cellular component classification of upregulated genes. (B) GO cellular component classification of downregulated genes. GO, gene ontology.

mellitus', 'arrhythmogenic right ventricular cardiomyopathy (ARVC)' and 'homologous recombination.

Interaction between differential expression of circRNAs and miRNAs. Endogenous circRNAs function as miRNA sponges, which means that the circRNAs bind miRNAs and repress their function $(13,18,25)$.

A total of 3,263 MREs of differentially expressed circRNAs were described bioinformatically using the Arraystar miRNA target prediction software. Analysis of the MRE sequence showed several binding sites between hsa_circRNA_103112 and various miRNAs. There were five binding sites between hsa_circRNA_103112 and hsa-miR-20b-3p, hsa-miR-93-3p, hsa-miR-370-3p, hsa-miR-520d-3p or hsa-miR-651-3p. The sequence of hsa_circRNA_103112 was complementary to: i) The miR-20b-3p seed region at the 5 ' end at position 61-66 with 7 mer-m 8 binding; ii) the miR-93-3p seed region at the 5 ' end of the nucleotide at position 142-147 with 7mer-m8 binding; iii) the miR-370-3p seed region at the $5^{\prime}$ end at position 143-148 with 8mer binding; iv) the miR-520d-3p seed region at the $5^{\prime}$ end at position $172-177$ with 7 mer-m8 binding; and v) the miR-651-3p seed region at the 5'end at position 112-117 with 8mer binding. This indicated that hsa_circRNA_103112 could function as a sponge for miR-20b-3p, miR-93-3p, miR-370-3p, miR-520d-3p and miR-651-3p (Fig. 6).

The MRE sequence and the target miRNA seed type (7mer-m8, 8mer) and 3'pairing sequence (nucleotides 13-16) are presented in Fig. 6. The precise base positions are shown in the alignments in the upper left and right corners. The seed type 7 mer-m8 shows an exact match to positions $2-8$ of the target miRNA (the seed + position 8 ), and 8 mer shows an exact match to positions $2-8$ of the target miRNA (the seed + position 8). Adenosine/uracil (A/U) content $30 \mathrm{nt}$ upstream and downstream of the seed sequence is shown in the column Local AU. Black bars represent guanine/cytosine and low accessibility whereas red bars represent $\mathrm{A} / \mathrm{U}$ and high accessibility of the seed. The height of the bars shows the extent of the accessibility. The position column presents the most likely relative MRE position on the linear presentation of the circRNA.

Interaction between differentially expressed circRNAs and $m R N A s$. circRNAs use their MRE to bind to miRNAs and consequently repress their function. Therefore, miRNAs can 


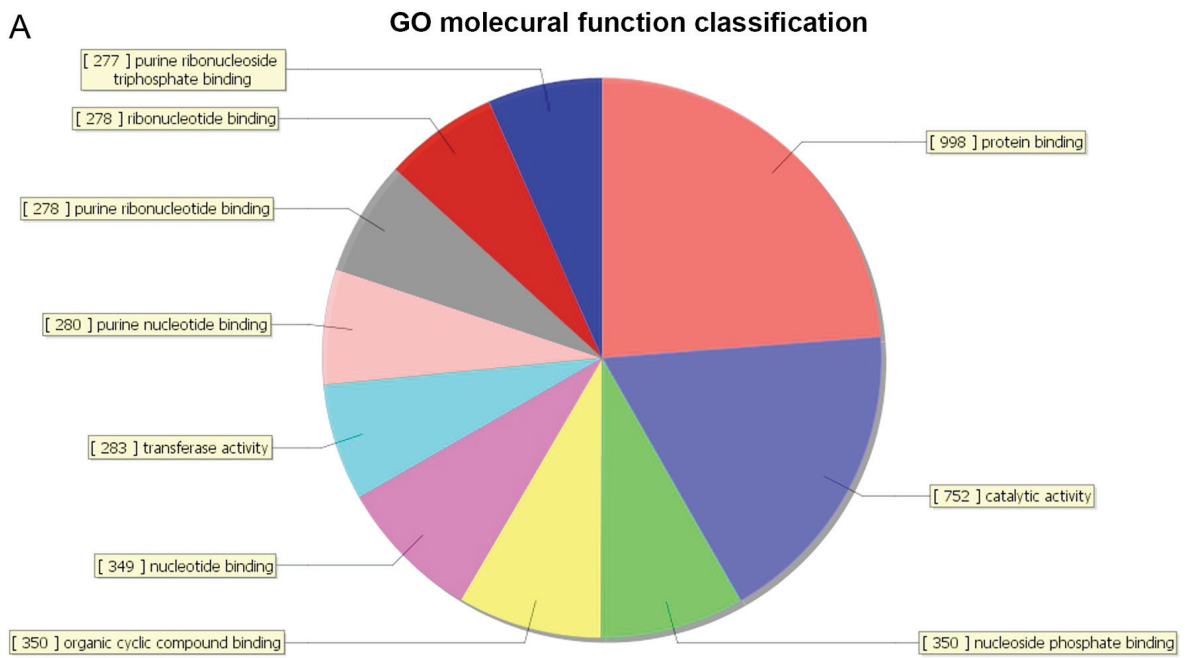

$\mathrm{B}$ GO molecural function classification

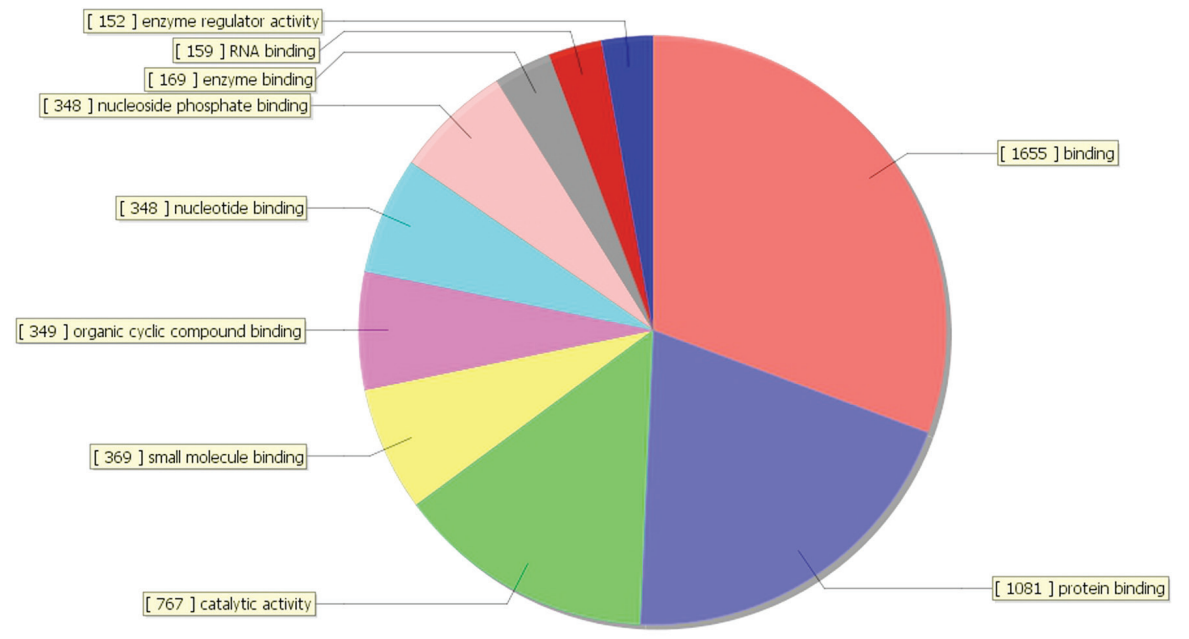

Figure 4. GO molecular function classification. (A) GO molecular function classification of upregulated genes. (B) GO molecular function classification of downregulated genes. GO, gene ontology.

identify the mRNA of a particular coding sequence by base complementary pairing, affecting the translation process and thus playing a role in regulating gene expression (32). The present study investigated the expression profiles of differentially expressed circRNA and mRNA and conducted an association analysis between circRNAs and mRNAs. A total of 254 circRNA transcripts were found in the corresponding differential mRNA expression profile information. Among the 254 circRNA associated with mRNA, there were 153 upregulated circRNAs and 101 downregulated circRNAs, indicating that these identical genes may regulate the occurrence and development of Down's syndrome through circRNA/miRNA/mRNA interactions. The results are presented in Fig. 7.

\section{Discussion}

At present, prenatal screening mainly in the early pregnancy and in the second trimester, ultrasound screening, maternal serum screening, and joint screening programs are used to assess the risk of fetal DS $(33,34)$. However, the false negative and false positive rates of traditional prenatal screening are relatively high (35), and invasive prenatal diagnosis methods such as amniocentesis, chorionic sampling and umbilical cord blood collection can easily lead to bleeding, infection, miscarriage and other complications (36). Therefore, it is necessary to identify novel biological markers in maternal blood to further improve the prenatal screening for DS during pregnancy.

Wu et al (37) reported that circRNAs may be used as biomarkers for the diagnosis of disease and evaluation of therapeutic efficacy. In addition, due to their high stability, circRNAs are easily obtained from body fluids $(10,18)$. Therefore, there has been increased interest in the in-depth and systematic research on circRNA. In view of the aforementioned study, under physiological conditions, the expression of circRNA in serum and plasma is relatively stable, while under pathological conditions; specific circRNA expression is likely to be abnormal.

The present study screened for differentially expressed circRNAs in umbilical cord blood obtained from pregnant women carrying fetuses with and without DS using circular gene chip technology. As a result, 735 differentially expressed circRNAs were detected, of which 414 were upregulated and 321 were downregulated. RT-qPCR was subsequently 
A

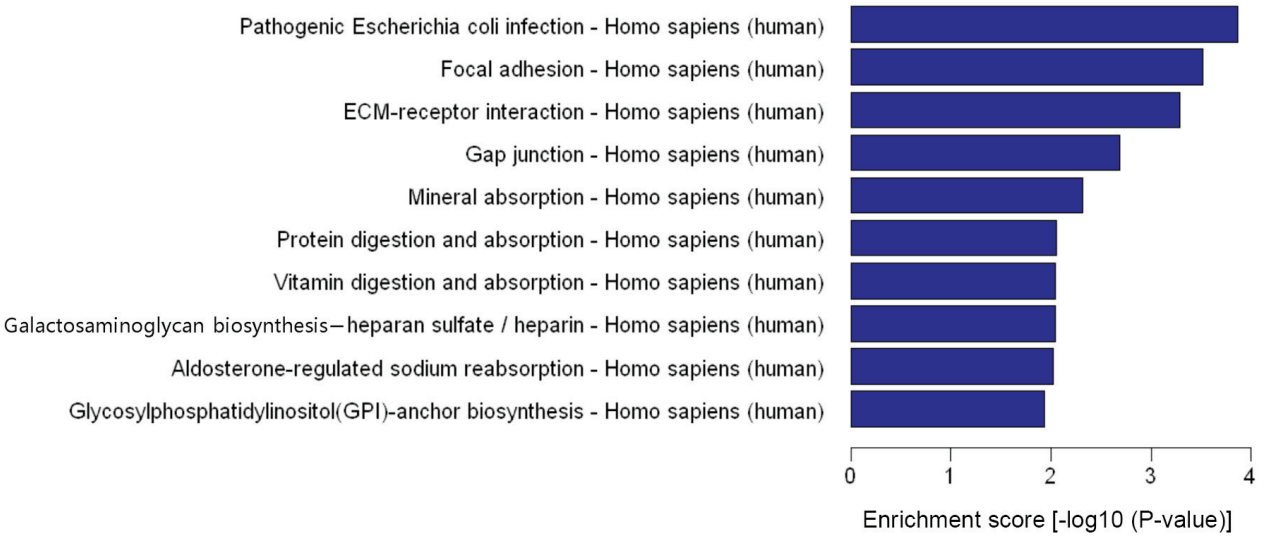

B

Differentially expressed genes

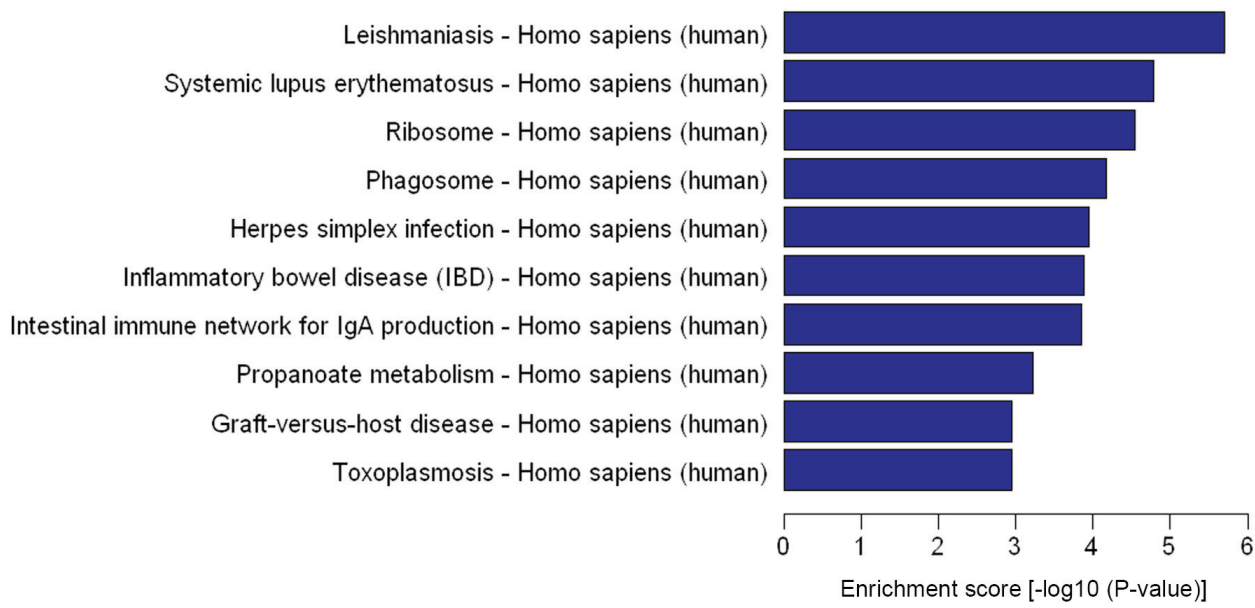

Figure 5. Significantly enrichment pathway analysis. (A) Differentially upregulated genes involved in the top ten pathways. (B) Differentially downregulated genes involved in the top ten pathways.

used to validate the differential expression of circRNA in peripheral blood samples obtained from children with and without DS. A total of six differentially expressed circRNAs, including three upregulated circRNAs (hsa_circRNA_103135, hsa_circRNA_103127 and hsa_circRNA_103112) and three downregulated circRNAs (hsa_circRNA_103137, hsa_circRNA_104907 and hsa_circRNA_101116) were selected for further analysis. Quantification using the $2^{-\Delta \Delta \mathrm{Cq}}$ method (29) revealed that the six circRNAs were differentially expressed between children with and without DS. There were extremely significant differences in hsa_circRNA_103127, hsa_circRNA_103112 and hsa_circRNA_104907 expression, but no significant differences in the expression of hsa_circRNA_103135, hsa_circRNA_103137 and hsa_circRNA_101116. These differences may be related to the temporal and tissue specificity of circRNA expression. Furthermore, analysis using Arraystar's miRNA target prediction software revealed that hsa_circRNA_103112 was associated with hsa-miR-20b-3p, hsa-miR-93-3p, hsa-miR-370-3p, hsa-miR-520d-3p and hsa-miR-651-3p.

Stamova et al (38) revealed that decreased miR-93-3p expression in the superior temporal sulcus (a region relevant to social interaction) and primary auditory cortex, and may have a potential impact on autism spectrum disorder (ASD). ASD is a neurodevelopmental disorder disease, the core symptoms of which include deficits in social communication and language, as well as ritual stereotyped behaviour, learning disability, immune system disturbances and sensory system alterations (39). Furthermore, Yanni et al (40) revealed that increased expression of endogenous miR-370-3p contributes to bradycardia associated with heart failure, suggesting that miR-370-3p may serve as a therapeutic target. The aforementioned phenotypic traits are often associated with DS $(41,42)$.

An Agilent expression profile chip was used to obtain the differentially expressed genes between pregnant women carrying fetuses with or without DS in the present study. A total of 6,619 differentially expressed genes were identified, of which 3,411 were upregulated and 3,208 were downregulated. Moreover, three genes (DYRK1A, USP25 and STRBP) corresponding to three significantly differentially expressed circRNAs (hsa_circRNA_103127, hsa_circRNA_103112 and hsa_circRNA_104907) were verified in peripheral blood samples obtained from children with and without DS. There was no significant difference in the expression level of DYRK1A and STRBP ( $\mathrm{P}>0.05)$. However, an extremely significant difference in USP25 expression was observed $(\mathrm{P}<0.01)$.

Valero et al (43) revealed that USP25 is a specific ubiquitin protease gene located in the $21 \mathrm{q} 11.2$ region, and that an increase in USP25 gene dosage in patients with DS 


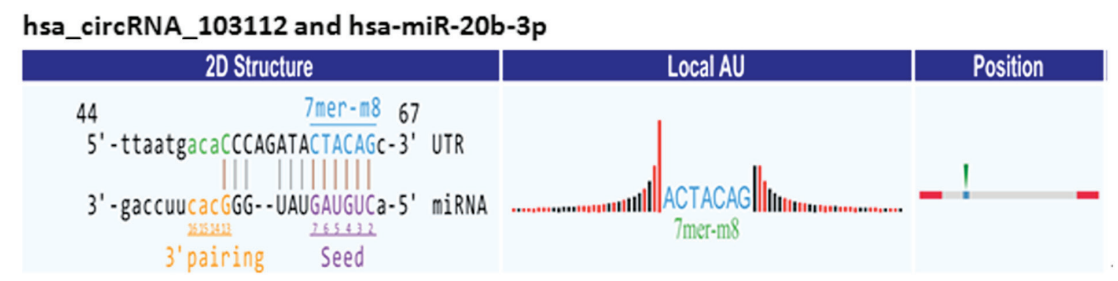

hsa_circRNA_103112 and hsa-miR-93-3p

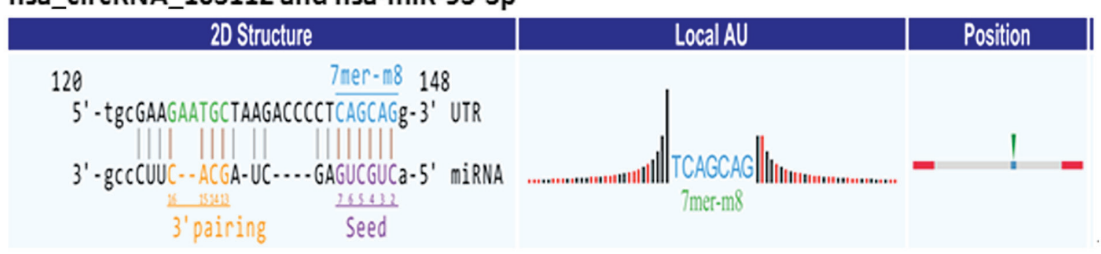

hsa_circRNA_103112 and hsa-miR-370-3p

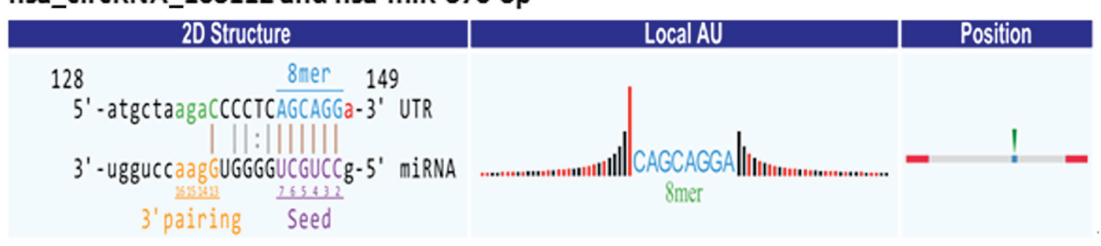

hsa_circRNA_103112 and hsa-miR-520d-3p

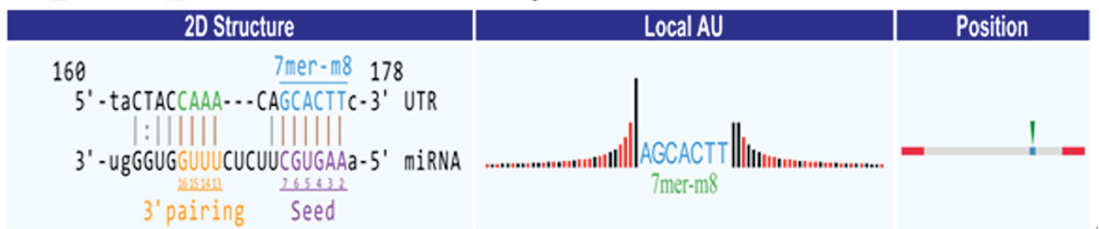

hsa_circRNA_103112 and hsa-miR-651-3p

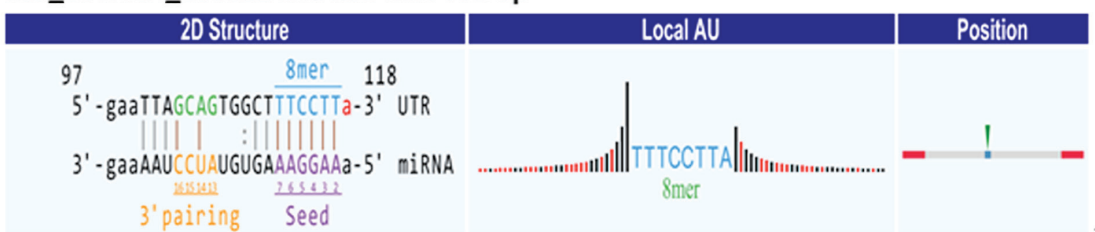

Figure 6. Results of target prediction and sequence analysis of miRNA response elements. miR/miRNA, microRNA; circRNA, circular RNA.

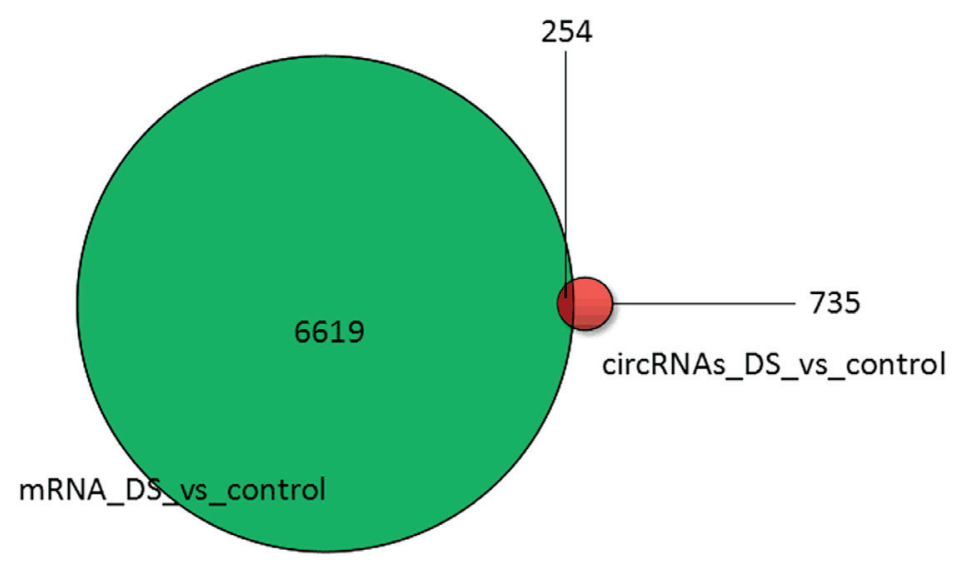

Figure 7. Common genes of related circRNA-mRNA. Transcripts of 254 differentially expressed circRNAs were also found to be differentially expressed in 6,619 mRNAs. A total of 254 of the 6,619 differentially expressed mRNAs corresponded to transcripts of differentially expressed circRNA. circRNA, circular RNA; DS, Down's syndrome.

disturbed the balance between ubiquitinated and deubiquitinated substrates. USP25 is a newly described member of the ubiquitin-specific processing proteases (UBPs) that encode the ubiquitin C-terminal hydrolases. Ubiquitin is a highly conserved eukaryotic protein (76 aa) and plays an important role in intracellular protein degradation. USP25 is associated with testis development and could be involved in the defective spermiogenesis found in males with DS (43). Increasing 
numbers of studies have found that disruption of ubiquitin systems can lead to a number of human diseases, including neurodegenerative disorders and several carcinomas (44-46). Valero et al (44) evaluated the expression level of USP25 in human fetal brains of DS and control disomic samples by RT-qPCR analysis and found an average 1.7-fold increase in USP25 expression in DS samples. Upregulation of USP25 in DS fetal brain indicates that it participates in the pathogenesis of DS and has the same gene dose effect as other UBP members associated with aneuploidy syndromes, such as ubiquitin specific peptidase 9 X-linked in Turner syndrome (47) and USP18 in DiGeorge syndrome (48).

The present study revealed that circRNA had an adsorption effect on miRNA, and the transcripts of differentially expressed circRNA were found in the corresponding differentially expressed gene expression profiles. A previous study demonstrated that miRNAs identify the mRNA of a particular coding sequence by complementary base pairing, affecting the translation process (49). Therefore, there is an interaction between circRNA/miRNA/mRNA, which may impact the occurrence and development of DS.

The present study, to best of our knowledge, was the first to reveal that circRNAs are differentially expressed between DS and control samples. The identified differentially expressed genes are mainly involved in cell division and development process and are also involved in primary immunodeficiency, rheumatoid arthritis and type I diabetes mellitus, as well as homologous recombination processes, and may result in abnormal copies of chromosome 21. This may disrupt normal development, causing patients with DS to experience developmental defects of the immune system and the emergence of various clinical symptoms (5). Most importantly, hsa_circRNA_103112 not only showed significant expression differences in cord blood, but also showed significant differences in the peripheral blood. Therefore, upregulatation of hsa_circRNA_103112 and its corresponding gene, USP25, in patients with DS patients may result in an expression imbalance of diploid genes through circRNA-miRNA-mRNA interactions and participates in the pathogenesis of DS. In addition, the present study revealed that the differential expression of hsa_circRNA_103112 in peripheral blood may serve as a novel diagnostic marker for the non-invasive prenatal screening for DS.

The present study had a number of limitations. Firstly, the study was limited by the small number of samples, which was not large enough to establish definitive conclusions. Furthermore, the samples were collected from Shenzhen People's Hospital and Guilin No. 924 Hospital, which may have resulted in regional differences. Secondly, the results may not be applicable to the general population. Thirdly, the study of circRNAs in DS is still in its initial stages, and the functional analysis requires further confirmation. Therefore, further circRNA expression studies with larger sample sizes, miRNA microarray analysis, RT-qPCR validation and circRNA knock-out or overexpression studies are required to further evaluate the potential of circRNAs in DS.

\section{Acknowledgements}

Not applicable.

\section{Funding}

The present study was supported by grants from the Guangxi Science and Technology Program (grant no. 1598012-25), Science and Technology Planning Project of Guangdong Province (grant no. 2017B020209001), Natural Science Foundation of Guangdong Province (grant no. 2017A030310629), Science and Technology Plan of Shenzhen (grant no. JCYJ20170307095606266) and Natural Science Foundation of Guangdong Province (grant no. 2016A020215027).

\section{Availability of data and materials}

The datasets used and/or analyzed during the current study are available from the corresponding author on reasonable request.

\section{Authors' contributions}

WS, DT and YD designed the study, analyzed the data and drafted the manuscript. YC, MO, JC, HL, WX, YW and $\mathrm{HH}$ acquired patient data and performed laboratory experiments. QG contributed to the study design and writing of the manuscript. All authors read and approved the final manuscript.

\section{Ethics approval and consent to participate}

The present study was approved by the Ethics Committee of Shenzhen People's Hospital (reference number, LL-KT-201801184) and the Ethics Committee of Guilin No. 924 Hospital. Written informed consent was obtained from all pregnant women and parents/guardians of the children.

\section{Patient consent for publication}

Not applicable.

\section{Competing interests}

The authors declare that they have no competing interests.

\section{References}

1. Kleschevnikov AM, Belichenko PV, Villar AJ, Epstein CJ, Malenka RC and Mobley WC: Hippocampal long-term potentiation suppressed by increased inhibition in the Ts65Dn mouse, a genetic model of down syndrome. J Neurosci 24: 8153-8160, 2004.

2. Roizen NJ and Patterson D: Down's syndrome. Lancet 361: 1281-1289, 2003.

3. Caughey AB, Washington AE and Kuppermann M: Perceived risk of prenatal diagnostic procedure-related miscarriage and down syndrome among pregnant women. Am J Obstet Gynecol 198: 333 e331-338, 2008.

4. Antonarakis SE, Lyle R, Dermitzakis ET, Reymond A and Deutsch S: Chromosome 21 and down syndrome: From genomics to pathophysiology. Nat Rev Genet 5: 725-738, 2004.

5. Thase ME: Longevity and mortality in down's syndrome. J Ment Defic Res 26: 177-192, 1982.

6. Cortés-López M, Gruner MR, Cooper DA, Gruner HN, Voda AI, van der Linden AM and Miura P: Global accumulation of circRNAs during aging in Caenorhabditis elegans. BMC Genomics 19: 8, 2018.

7. Chen LL and Yang L: Regulation of circRNA biogenesis. RNA Biol 12: 381-388, 2015. 
8. Salomon LJ, Alfirevic Z, Audibert F, Kagan KO, Paladini D, Yeo G and Raine-Fenning N; ISUOG Clinical Standards Committee: ISUOG updated consensus statement on the impact of cfDNA aneuploidy testing on screening policies and prenatal ultrasound practice. Ultrasound Obstet Gynecol 49: 815-816, 2017.

9. Houseley JM, Garcia-Casado Z, Pascual M, Paricio N, O'Dell KM, Monckton DG and Artero RD: Noncanonical RNAs from transcripts of the Drosophila muscleblind gene. J Hered 97: 253-260, 2006.

10. Salzman J, Gawad C, Wang PL, Lacayo N and Brown PO Circular RNAs are the predominant transcript isoform from hundreds of human genes in diverse cell types. PLoS One 7: e30733, 2012.

11. Salzman J, Chen RE, Olsen MN, Wang PL and Brown PO: Cell-type specific features of circular RNA expression. PLoS Genet 9: e1003777, 2013.

12. Jeck WR, Sorrentino JA, Wang K, Slevin MK, Burd CE, Liu J, Marzluff WF and Sharpless NE: Circular RNAs are abundant, conserved, and associated with ALU repeats. RNA 19: 141-157, 2013.

13. Memczak S, Jens M, Elefsinioti A, Torti F, Krueger J, Rybak A, Maier L, Mackowiak SD, Gregersen LH, Munschauer M, et al: Circular RNAs are a large class of animal RNAs with regulatory potency. Nature 495: 333-338, 2013.

14. Zhang Y, Zhang XO, Chen T, Xiang JF, Yin QF, Xing YH, Zhu S, Yang L and Chen LL: Circular intronic long noncoding RNAs. Mol Cell 51: 792-806, 2013.

15. Guo JU, Agarwal V, Guo H and Bartel DP: Expanded identification and characterization of mammalian circular RNAs. Genome Biol 15: 409, 2014

16. Li Z, Huang C, Bao C, Chen L, Lin M, Wang X, Zhong G, Yu B, Hu W, Dai L, et al: Exon-intron circular RNAs regulate transcription in the nucleus. Nat Struct Mol Biol 22: 256-264, 2015.

17. Valdmanis PN and Kay MA: The expanding repertoire of circular RNAs. Mol Ther 21: 1112-1114, 2013.

18. Hansen TB, Jensen TI, Clausen BH, Bramsen JB, Finsen B, Damgaard CK and Kjems J: Natural RNA circles function as efficient microRNA sponges. Nature 495: 384-388, 2013.

19. Kaikkonen MU, Lam MT and Glass CK: Non-coding RNAs as regulators of gene expression and epigenetics. Cardiovasc Res 90: 430-440, 2011.

20. Chu T, Mouillet JF, Hood BL, Conrads TP and Sadovsky Y: The assembly of miRNA-mRNA-protein regulatory networks using high-throughput expression data. Bioinformatics 31: 1780-1787, 2015.

21. Chen CY and Sarnow P: Initiation of protein synthesis by the eukaryotic translational apparatus on circular RNAs. Science 268: 415-417, 1995.

22. Perriman R and Ares M Jr: Circular mRNA can direct translation of extremely long repeating-sequence proteins in vivo. RNA 4: 1047-1054, 1998.

23. Hentze MW and Preiss T: Circular RNAs: Splicing's enigma variations. EMBO J 32: 923-925, 2013.

24. Bohjanen PR, Colvin RA, Puttaraju M, Been MD and Garcia-Blanco MA: A small circular TAR RNA decoy specifically inhibits Tat-activated HIV-1 transcription. Nucleic Acids Res 24: 3733-3738, 1996.

25. Hansen TB, Kjems J and Damgaard CK: Circular RNA and miR-7 in cancer. Cancer Res 73: 5609-5612, 2013.

26. Li Y, Zheng Q, Bao C, Li S, Guo W, Zhao J, Chen D, Gu J, He X and Huang S: Circular RNA is enriched and stable in exosomes: A promising biomarker for cancer diagnosis. Cell Res 25: 981-984, 2015.

27. Sun H, Tang W, Rong D, Jin H, Fu K, Zhang W, Liu Z, Cao H and Cao X: Hsa_circ_0000520, a potential new circular RNA biomarker, is involved in gastric carcinoma. Cancer Biomark 21: 299-306, 2018.

28. Yang Y, Gao X, Zhang M, Yan S, Sun C, Xiao F, Huang N, Yang X, Zhao K, Zhou H, et al: Novel role of FBXW7 Circular RNA in repressing glioma tumorigenesis. J Natl Cancer Inst 110: 304-315, 2018.

29. Livak KJ and Schmittgen TD: Analysis of relative gene expression data using real-time quantitative PCR and the 2(-Delta Delta C(T)) method. Methods 25: 402-408, 2001.

30. Enright AJ, John B, Gaul U, Tuschl T, Sander C and Marks DS: MicroRNA targets in drosophila. Genome Biol 5: R1, 2003.

31. Pasquinelli AE: MicroRNAs and their targets: Recognition, regulation and an emerging reciprocal relationship. Nat Rev Genet 13: 271-282, 2012.
32. Lawrie CH: MicroRNAs and haematology: Small molecules, big function. Br J Haematol 137: 503-512, 2007.

33. Yan-Liang D and Xiao-Nan Y: Combined serum and ultrasound screening for early diagnosis of Down's syndrome. Chin J Prac Gynecol Obstetrics 26: 895-898, 2010.

34. Benn PA, Kaminsky LM, Ying J, Borgida AF and Egan JF: Combined second trimester biochemical and ultrasound screening for Down syndrome. Obstet Gynecol 100: 1168-1176, 2001.

35. Langlois S and Brock JA; Genetics Committee: Current status in non-invasive prenatal detection of down syndrome, trisomy 18 , and trisomy 13 using cell-free DNA in maternal plasma. J Obstet Gynaecol Can 35: 177-181, 2013 (In English, French).

36. Kolker A and BurkeBM: Grieving the wanted child: Ramifications of abortion after prenatal diagnosis of abnormality. Health Care for Women Int 14: 513-526, 1993.

37. Wu Q, Wang Y, Cao M, Pantaleo V, Burgyan J, Li WX and Ding SW: Homology-independent discovery of replicating pathogenic circular RNAs by deep sequencing and a new computational algorithm. Proc Natl Acad Sci USA 109: 3938-3943, 2012.

38. Stamova B, Ander BP, Barger N, Sharp FR and Schumann CM: Specific regional and age-related small Noncoding RNA expression patterns within superior temporal gyrus of typical human brains are less distinct in autism brains. J Child Neurol 30: 1930-1946, 2015.

39. Lo YC, Chen YJ, Hsu YC, Tseng WI and Gau SS: Reduced tract integrity of the model for social communication is a neural substrate of social communication deficits in autism spectrum disorder. J Child Psychol Psychiatry 58: 576-585, 2017.

40. Yanni J,ZiM, Choudhury M,Cai X,Logantha S,Li J,CartwrightE, Dobrzynski H, Hart G and Boyett MR: MicroRNA 370-3p could explain the dysfunction of the cardiac conduction system in heart failure. Proc Physiol Soc 34: PC158, 2015.

41. Warner G, Howlin P, Salomone E, Moss J and Charman T: Profiles of children with Down syndrome who meet screening criteria for autism spectrum disorder (ASD): A comparison with children diagnosed with ASD attending specialist schools. J Intellect Disabil Res Jidr 61: 75-82, 2017.

42. Levine OR and Simpser M: Alveolar hypoventilation and cor pulmonale associated with chronic airway obstruction in infants with down syndrome. Clin Pediatr (Phila) 21: 25-29, 1982.

43. Valero R,Marfany G, González-Angulo O,González-González G, Puelles L and Gonzàlez-Duarte R: USP25, a novel gene encoding a deubiquitinating enzyme, is located in the gene-poor region 21q11.2. Genomics 62: 395-405, 1999.

44. Valero R, Bayés M, Francisca Sánchez-Font M, González-Angulo O, Gonzàlez-Duarte R and Marfany G: Characterization of alternatively spliced products and tissue-specific isoforms of USP28 and USP25. Genome Biol 2: RESEARCH0043, 2001.

45. Ciechanover A and Brundin P: The ubiquitin proteasome system in neurodegenerative diseases: Sometimes the chicken, sometimes the egg. Neuron 40: 427-446, 2003.

46. Hershko D, Bornstein G, Ben-Izhak O, Carrano A, Pagano M, Krausz MM and Hershko A: Inverse relation between levels of p27Kip1 and of its ubiquitin ligase subunit Skp2 in colorectal carcinomas. Cancer 91: 1745-1751, 2001.

47. Jones MH, Furlong RA, Burkin H, Chalmers IJ, Brown GM, Khwaja O and Affara N: The drosophila developmental gene fat facets has a human homologue in Xp11.4 which escapes $\mathrm{X}$-inactivation and has related sequences on Yq11.2. Hum Mol Genet 5: 1695-1701, 1996.

48. Schwer H, Liu LQ, Zhou L, Little MT, Pan Z, Hetherington CJ and Zhang DE: Cloning and characterization of a novel human ubiquitin-specific protease, a homologue of murine UBP43 (USP18). Genomics 65: 44-52, 2000.

49. Dalmay T: Mechanism of miRNA-mediated repression of mRNA translation. Essays Biochem 54: 29-38, 2013.

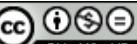

This work is licensed under a Creative Commons Attribution-NonCommercial-NoDerivatives 4.0 International (CC BY-NC-ND 4.0) License. 